\title{
Hans Magnus Enzensberger, Reiner Kunze. Traductions et imitations : la fracture du trait
}

Hans Magnus Enzensberger, Reiner Kunze. Übersetzungen und Imitationen: ein «Ruf zum Aufbruch»

Hans Magnus Enzensberger, Reiner Kunze - Translations and Imitations:

Breaking through

\section{Béatrice Poulain}

\section{OpenEdition}

Journals

Édition électronique

URL : http://journals.openedition.org/ceg/368

DOI : $10.4000 /$ ceg.368

ISSN : 2605-8359

\section{Éditeur}

Presses Universitaires de Provence

Édition imprimée

Date de publication : 5 avril 2017

Pagination : 103-120

ISBN : 979-10-320-0103-5

ISSN : 0751-4239

\section{Référence électronique}

Béatrice Poulain, «Hans Magnus Enzensberger, Reiner Kunze. Traductions et imitations : la fracture du trait », Cahiers d'Études Germaniques [En ligne], 72 | 2017, mis en ligne le 05 octobre 2018, consulté le 26 novembre 2020. URL : http://journals.openedition.org/ceg/368 ; DOI : https://doi.org/10.4000/ ceg.368 


\title{
Hans Magnus Enzensberger, Reiner Kunze
}

\author{
Traductions et imitations : la fracture du trait
}

\author{
Béatrice POULAIN \\ Université Paris 3 Sorbonne-Nouvelle
}

Entre la rupture, la brisure, les angles et zigzags d'une ligne résolument réfractaire qui pourrait rappeler le gothique mais dont on ne sait plus finalement quelle langue elle dessine, quels signes? S'agit-il encore de mots ou n'en subsiste-t-il plus que la trace, des réfractions réfléchissantes parcourant un espace ouvert par effraction où apparaissent les ombres de portraits fractionnés?

Né de la même racine indo-européenne que le verbe latin frangere, fregi, fractum, au cœur de cette variation étymologique délimitant notre espace de réflexion, l'allemand brechen permet un jeu thématique analogue entre la violence de la fragmentation et l'ouverture réfléchissante qui en découle: Bruch, Brechungen, Aufbrechen, Durchbrechen, Zerbrechen, Bruchstück, Verbrecher et aussi Aufbruch. Rapporté à la traduction, le thème de la fracture s'avère des plus riches dans son ambivalence même. Son articulation allemande, particulièrement évocatrice, reviendra hanter ponctuellement cette étude consacrée en grande partie à Hans Magnus Enzensberger et à son anthologie poétique de 1998, Geisterstimmen, dont le sous-titre: "Übersetzungen und Imitationen " suscite intérêt et interrogations ${ }^{1}$.

En effet, ce sous-titre souligne d'emblée le caractère " autre " de cette anthologie dont la dimension personnelle et active reprend et varie deux vastes anthologies précédentes, Museum der modernen Poesie (1960) ${ }^{2}$ et Wasserzeichen $(1985)^{3}$. On y retrouve certains poèmes déjà traduits pour le Musée où Enzensberger cherchait à " instituer " non en " mausolée " mais en " annexe d'atelier " (Annex zum Atelier) le caractère classique d'une poésie moderne (1910-1945). Les « Imitations " regroupent, elles, la plupart des poèmes pastichés par Enzensberger sous un autre nom dans l'anthologie ludique et érudite Wasserzeichen. Éditée sous le pseudonyme d'Andreas Thalmayr, celle-ci présentait en cent soixante-quatre variantes (Spielarten) " l'art et le plaisir de

1. Hans Magnus Enzensberger (H.M.E.), Geisterstimmen, Frankfurt am Main, Suhrkamp, 1999.

2. Museum der modernen Poesie, eingerichtet von H.M.E., Frankfurt am Main, Suhrkamp, 1960 ( citations dans la version poche, 2 volumes, 1980).

3. Das Wasserzeichen der Poesie. Oder die Kunst und das Vergnügen Gedichte zu lesen. In hundertvierundsechzig Spielarten vorgestellt von Andreas Thalmayr, édité par H.M.E., Nördlingen, Franz Greno (coll. « Die andere Bibliothek »), 1985. 
lire des poèmes ". Son enjeu: renouveler le regard du lecteur sur une poésie trop souvent perçue comme empoussiérée et ennuyeuse et non comme aire de jeux divertissante où poète et lecteur s'amuseraient en commun.

Les liens étroits entre lecture et écriture sont donc une nouvelle fois prédominants quand Enzensberger décide en 1998, non de rééditer, comme en 1979, le Musée de $1960^{4}$, mais de créer une anthologie personnelle de poèmes qui sont toujours aussi de sa plume - de traducteur ou d'imitateur. Le Post-Scriptum de ce qui se présente alors comme une longue lettre au lecteur, souligne la dimension purement subjective du critère de sélection: n'ont été retenus par Enzensberger que les textes lui "plaisant aujourd'hui encore ". On est loin de la préface du Musée, d'une légitimation théorique. Comme dans Wasserzeichen en revanche, nul canon "des plus beaux, des plus importants, des meilleurs " poèmes ${ }^{5}$. À l'image des traductions, issues de ses " préférences et idiosyncrasies " personnelles ${ }^{6}$, les imitations, nous précise encore Enzensberger, sont des hommages aux "prédécesseurs et compagnons de lutte " qui lui ont tout appris du poème : n'ont fait l'objet de "sacrilège " que les poètes " pris au sérieux ", " de même que le blasphème ne signifie quelque chose qu'au croyant ${ }^{7}$ ".

Si l'anthologie est bien personnelle, quel en est l'enjeu? Pourquoi avoir voulu conjuguer le Musée et les Wasserzeichen? Pourquoi ce montage si personnel qu'il en devient, je cite, un portrait indirect, une "image reflétée " (Spiegelbild), de l'auteur ${ }^{8}$, avec moult excroissances subjectives? Cette déformation s'approfondit dans le rapport entre les deux parties du recueil, totalement disproportionnées dans la forme et inclassables dans le contenu. Après cent soixante-quatre traductions sont proposées vingt-six imitations. Et pourquoi dans cet ordre? Pourquoi la rupture, dans chacune des parties, d'un ordre chronologique présenté comme règle ${ }^{9}$ ? Comment se fait-il que le modèle original des imitations soit parfois présent, parfois non, et toujours absent pour les traductions? La relation entre traductions et imitations laisse sur bien des points perplexe. Les imitations ne se résument pas à la possibilité d'inclure, dans cette longue déclaration d'amour, des poètes allemands puisque douze des poètes imités sont d'origine étrangère. Inversement, la présence de modèles allemands n'autorise pas à lire en ces dernières un simple débordement impertinent de la traduction, d'autant plus que certaines imitations, celles d'Edward Lear notamment, ont déjà été publiées comme recueil de traductions. Enzensberger, lui, ne souligne dans le post-scriptum que le rapport de gradation entre traduction et imitation. Quand

4. On notera l'intervalle de dix-neuf ans qui sépare chaque réédition de la précédente.

5. L'ensemble des poèmes présente en lui-même excroissances et maigreurs dignes du plus beau miroir déformant (ainsi un poème d'Ungaretti, dix-neuf poèmes de César Vallejo) : en ce sens, on pourrait presque y reconnaître une parodie d'anthologie.

6. H.M.E., Geisterstimmen, p. 392.

7. Ibid., p. 384 : « ... erst im Sakrileg zeigt sich, was einer ernst nimmt, so wie die Blasphemie nur dem Frommen etwas bedeutet ".

8. Ibid., p. 392: "Was dabei im Lauf der Zeit entsteht, ist eher eine Art Collage als ein Kanon; vielleicht sogar ein Spiegelbild, in dem man das Selbstportrait eines Dichters erblicken kann. "

9. Ibid., p. 371. 
" absence de scrupules " et prise de libertés s'intensifient, alors la traduction " frise " (grenzt an) la paraphrase, l'imitation. Le geste est fondamentalement le même: "sacrilège ". Son auteur: un " contrebandier " au "rire froid ". Bruch et Verbrechen, fracture et effraction criminelles, iconoclastes, rendent le texte "vivant " (beleben) : ces professions de foi larvées " où se montre ce que quelqu'un prend au sérieux " pourraient s'avérer conditions de transmission réelle des textes, du savoir poétique d'Enzensberger, de ce qui lui serait poétiquement essentiel.

Pour mieux saisir un dispositif où tout est fait pour déjouer les regards, se rire de l'analyse, un détour par l'autre du même s'avère fructueux: la limpidité de Reiner Kunze dans son évocation de la Nachdichtung lors d'un cycle de conférences d'esthétique de la fin des années $1980^{10}$, l'inaltérable clarté de ces écrits unissant eux aussi une approche explicitement personnelle et la citation de poèmes traduits en hommage à la lutte qui s'y exprime, ouvrent des brèches souriantes, permettent des aperçus lumineux sur ce qui se joue dans l'obscurité, derrière l'hilarité du masque, chez Enzensberger.

L'approche se fera en trois temps et en une variation étymologique sur la fracture et le Bruch allemand :

I) La traduction comme destruction et recomposition / Nachdichtung als Zerbrechen und Gliedertausch

II) L'imitation comme effraction: quand le fléau de la familiarité invite à la distanciation / Imitation als Heimbruch: wie die Heimsuchung zur Verfremdung ruft

III) CONCLUSION :

Faut-il rompre la promesse du bonheur? Du politique à l'utopique, le grand sérieux du rire

\section{La traduction comme destruction et recomposition / Nachdichtung als Zerbrechen und Gliedertausch}

Dasselbe, das ein anderes ist - Unter Nachdichten verstehe ich, ein Gedicht so zu übersetzen, $\mathrm{da} ß$ es in der Sprache, in die es übersetzt wird, wie ein Original wirkt, und daß dieses dem fremdsprachlichen Original höchstmöglich gleicht. Nachdichten heißt, dasselbe zu schaffen, das ein anderes ist - ein Eigenes, das ein Fremdes bleiben muß. ${ }^{11}$

La défınition par laquelle Reiner Kunze débute sa conférence sur la traduction s'adosse à la conférence précédente, dédiée à l'image et à l'expérience poétiques. L'effet de " l'original ", c'est-à-dire ici du poème en langue originale, s'y donnait à lire comme répétition, chez le lecteur, d'une irruption ou d'un surgissement initial, d'un étonnement premier, vécu par le poète, avant de se transmuer, sous

10. Reiner Kunze, "Konsequenzen des Ästhetischen. Münchner Poetik Vorlesungen ", in Das weiße Gedicht, Frankfurt am Main, Fischer Verlag, 1989, p. 9-117.

11. Reiner Kunze, "Dasselbe, das ein anderes ist. Über das Nachdichten ", in Das weiße Gedicht, p. 65. 
sa plume, en mots: "Der poetische Einfall, der aus dem Unbewussten aufsteigt, überrascht den Dichter ebenso wie das aus dem Einfall hervorgegangene dichterische Bild den Leser. "

Au moment où, selon les mots d'Octavio Paz, cité à l'appui par Kunze, le lecteur entre dans " le champ électrique " que génère la " puissance de révélation " du poème, quand, en d'autres termes, il " transperce le mur du temps "(Durchbrechen), sa lecture devient création seconde, Nachschöpfung pour Kunze, Wiedererschaffung pour Paz. L'image poétique seconde (Abbild) du lecteur différera nécessairement de celle du poète, mais " l'acte de création lui-même " est, nous dit Paz, totalement identique ${ }^{12}$.

Quand Kunze écrit qu'un poème traduit sur le mode de la Nachdichtung " agit comme un original ", nous en sommes à la quatrième répétition de l'acte d'écriture issu d'un surgissement initial ${ }^{13}$. Écriture, lecture, traduction sont d'entrée de jeu identifiables comme imitations et transmissions d'une expérience initiale d'irruption, d'une rupture de la maîtrise exercée par la ratio sur les choses ${ }^{14}$. L'imitation apparaît aisément comme répétition d'un processus, qui est le processus poétique lui-même, et non comme reproduction d'un modèle.

L'original est ici intimement lié à l'origine, au surgissement comme impulsion créatrice. La traduction la plus ressemblante, aux yeux de Kunze, sera celle qui sonnera le plus originel, le plus allemand: elle sera profondément originale. L'exemple de traduction alors citée en modèle par Kunze est ainsi, à première vue, confondant d'irressemblance. Il s'agit d'un poème tchèque de Jaroslav Seifert traduit en allemand par H. C. Artmann: " Friedhof in Genua ".

Original

Dva pristavy

O Janovane

More se vsdouva

A neustane

Zivot a more Zivot a more
Traduction littérale

Zwei Häfen

0 Genuese

Die See geht hoch

Und wird nicht innehalten

Leben und Meer Leben und Meer
Traduction H.C. Artmann

Zwei häfen

ihr Genuesen

an land ist noch

keiner genesen

Seemanslos Seemanslos

Selon Kunze, ces vers sont à la fois " allemands " et " les plus fidèles qu'il soit " à l'original. Seifert lui-même se serait exclamé: " c'est mieux que l'original!" . Kunze légitime théoriquement son avis: la rime, Genuesen-genesen, inédite en

12. Reiner Kunze, " Die poetische Vorstellung ", p. 59-60. Les citations d'0. Paz sont issues du recueil Der Bogen und die Leier, Frankfurt am Main, Suhrkamp, 1983 (version espagnole 1956).

13. Cf. 0. Paz : " Le poème est la métaphore de ce qu'a senti et pensé le poète. Cette métaphore est la résurrection de l'expérience et sa transmutation. La lecture du poème reproduit ce double mouvement de changement et de résurrection. La traduction poétique, à son tour, répète la même opération, quoique de façon plus radicale encore: elle ne cherche pas l'impossible identité mais la difficile ressemblance. ", in Lecture et contemplation, Paris, éd. La Délirante, 1982, p. 18.

14. Du surgissement de "l'inconscient " naît une " image poétique " (dichterisches Bild) dont " la tension entre fascination (Hingerissensein) et irritation " (Kunze, "Die poetische Vorstellung ", p. 59) - face à l'in-compréhensible échappant au concept - suscite à nouveau le " développement " de l'exclamation initiale qui, pour Paul Valéry, constitue le poème. 
allemand, est préservée et maintenue à sa place, et le soupir du marin est une " performance " (Glanzleistung). Il n'entre pas dans le détail, mais les points qu'il développe ensuite en s'aidant de ses propres traductions, permettent de comprendre pourquoi : il y a certes fidélité au sens général, émergeant derrière les mots, mais aussi à l'ordre du rythme et de sa vocalisation: janovaneneustane/genuesen-genesen. On retrouve une rime riche (ane-esen) avec longue-brève (en " e "), un rythme 4-3 et une allitération en " n ". Pour Zivota more/seemannslos, le rythme vocalisé zi-a-mo, devient ze-ma-o, longue-brèvelongue, donc extrêmement proche du tchèque.

Le processus ou la procédure de la traduction apparaît immédiatement comme un jeu où le va-et-vient entre le sens et la matière du mot, de cette " formation de mots " (Wortgebilde), comme dit Gadamer ${ }^{15}$, qu'est le poème, distend, distord, dissèque joyeusement ${ }^{16}$, crée des espaces où se dessine la texture intime des rapports entre son et sens et d'où pourra surgir une autre constellation poétique, à la fois la même et une autre. À chaque traduction, la question de la fidélité et du nécessaire écart se pose autrement. Dans un cas, il s'agira de préserver un noyau intangible tout en veillant à éviter un néologisme superflu ${ }^{17}$. La traduction d'un " mot créé " (Kunstwort) requerra, elle, l'élaboration d'un équivalent allemand aux résonances similaires. Dans " Herbst hinter der Stadt ", Jan Skacel " confronte ", selon Kunze, " ceux qui affirment que l'homme peut tout expliquer, à un Kunstwort qui n'est pas totalement explicable ". Mot à mot: " und was ist zatopoli? ". La traduction exige alors un " démontage " (Zerlegen) du concept en préfixe-racine-suffıxe et une recomposition qui préfèrera obéir à la tonalité de calme et de tendre sécurité (Geborgenheit) plutôt qu'à l'exactitude sémantique: um-pappel-sein deviendra umlindensein dans un allemand culturel où résonnent chants et poèmes (Am Brunnen vor dem Tore, ou le Lied de Mahler, Auf der Straße steht ein Lindenbaum).

Entre " démontage " et répétition créatrice, la traduction poétique se fait alors " échange de membres " (Gliedertausch), selon la métaphore de Celan reprise et développée par Kunze dans son journal de 1992 Am Sonnenhang:

der Name Ossip kommt auf dich zu, du erzählst ihm, was er schon weiß, er nimmt es, nimmt es dir ab, mit Händen, du löst ihm den Arm von der Schulter, den rechten, den linken, du heftest die deinen an ihre Stelle... $\quad \rightarrow \quad$ Kunze: meinen Vers für seinen Vers

15. Hans-Georg Gadamer, Die Aktualität des Schönen, Stuttgart, Reclam, 1977, p. 55.

16. Cette joie peut s'exprimer par un recours aux couleurs faisant ressortir assonances, allitérations et rimes, dès lors qu'à une voyelle correspond une couleur précise, par exemple le marron au " o ". Reiner Kunze évoque cette technique qu'il partage avec sa traductrice française Rose-Marie François dans son journal Am Sonnenhang, Frankfurt am Main, Fischer Verlag, 1995, p. 95.

17. Ainsi, le jeu assourdissant des rapports entre le matin, sa rosée et ses grenouilles, qui en tchèque se retrouvent dans la racine " ros ", se jouera en allemand sur le son " au ", de "Laubfrosch " et de " Tau ", mais aussi de " laut " et de " taub " : le poème de Jan Skacel trouve en allemand une variante feuillue, qui évite un néologisme exotique (Taufrosch) mais préserve un noyau intangible traversant indemne le fleuve entre les langues : " für alle die im herzen barfuß sind". 
- was abriß, wächst wieder zusammen

er nimmt auch das, und du hast wieder, was dein ist, was sein war...
- $\rightarrow$ Kunze: übersetzen heißt verwunden, um zusammenwachsen lassen zu können

$\rightarrow$ Kunze: Das, was der Übersetzer an eigener poetischer Erfindung hingibt, verdankt er dem Original, das ihn inspirierte. ${ }^{18}$

Sa fidélité à l'original conduira même le traducteur à opter pour la paraphrase lorsque le poème vit davantage de ses jeux de rimes et d'assonances que du sens qui s'en dégage. "Ce qu'il s'agit alors de traduire ", nous dit Kunze, " c'est le plaisir de ce qui fait plaisir " (der Spaß am Spaß). Sujet et sonorités générales inspirent une " adaptation ", un texte allemand similaire, c'est-à-dire tout aussi absurde (Nonsenstext) et joueur que l'original. L'auteur propose alors en opposition à la traduction littérale, " informative ", d'un poème de Vaclav Čtvretek une traduction à la fois plus ludique, plus lointaine, et plus proche $d u$ rapport au texte induit par l'original :

Au mot à mot

In einem Meer am Äquator

Verschluckte ein Hai eine Ziehharmonika,

Ein Walfisch, der morgens immer

Am Piano saß,

Sagte: Hallo, Hai,

Laß uns zum Tanz aufspielen!

\section{Traduction de Kunze}

Ein Hai fraß eine Orgel, die saß ihm in der Gorgel, die saß ihm in der Gurgel, und klang wie eine Urgel. Der Wal schlug aufs Klavier, das klang, als spielten vier.

Cet exemple de texte absurde est particulièrement instructif. En effet, le recueil d'Edward Lear traduit par Enzensberger s'intitule justement Nonsense Songs and Stories. La traduction, "Edward Lears Kompletter Nonsens ", est si réussie que le poète et traducteur Joachim Sartorius l'évoque d'entrée de jeu dans un article dédié à " Enzensberger comme médiateur " ou passeur (Vermittler), saluant au passage les qualités du traducteur: " höchste Findigkeit, grenzenlose Bedenkenlosigkeit ${ }^{19}$ ".

Or, dans Geisterstimmen, les traductions de Lear comptent parmi les Imitations, sous l'appellation de "paraphrase " tandis que les traductions d'Edward Wallace ou de Charles Simic, ensuite évoquées par Sartorius, font, elles, bien partie des "Traductions ", sans qu'aucune distinction extérieure ne puisse être observée sur le plan de la fidélité à l'original ${ }^{20}$. Soulignons que le processus de traduction répond chez Enzensberger en tous points aux approches de Reiner Kunze. Un bref regard sur quelques vers traduits d'Apollinaire par Enzensberger suffit pour s'en convaincre. Dans Zone, la rime " rentrer à pied- d'Océanie et de Guinée "

18. Kunze, Am Sonnenhang, p. 105.

19. Joachim Sartorius, "Schmuggler, Torwächter, Virtuose ", in Der Zorn altert, die Ironie ist unsterblich. Über Hans Magnus Enzensberger, Frankfurt am Main, Suhrkamp taschenbuch, 1999, p. 204.

20. On en prendra pour preuve l'exemple de " Frightening Toys " cité par Sartorius (ibid, p. 206) : " History practising its scissor-clips / In the dark / So everything comes out in the end / Missing an arm or a leg ". Dans la traduction d'H.M.E.: " Die Geschichte probiert ihre Schere / im Dunkeln aus, so / daß am Ende allem und jedem / ein Arm fehlt oder ein Bein ". 
se métamorphose en "nach Haus um zu ruhn - aus Insulinde und Kamerun ". À première vue, la traduction est très éloignée de l'original. L'approche de Kunze œuvrant par démembrement et reconstruction permet cependant de reconstituer les échos et analogies sur lesquels se fonde la traduction. La rime intérieure " pied "-« Guinée " se muant en " ruhn "-« Kamerun " en représente la clef de voûte. Le " s " d'Océanie se retrouve ensuite dans Insulinde, de même que les deux " i " d'Océanie et de Guinée. Les deux " $\mathrm{n}$ " sont préservés, le " a " migre vers le "Kamerun ", tandis que les deux noms exotiques restent rythmiquement liés par une assonance non plus en " e " mais en " u " ${ }^{21}$. L'équilibre général, le mouvement et l'exotisme restent préservés.

Les processus de traduction sont donc ceux mêmes du Gliedertausch. Dans l'avant-propos de Wasserzeichen, puis dans le post-scriptum des Geisterstimmen, cette lecture qu'est la traduction revient, selon Enzensberger lui-même, précisément à une " destruction " (zerstören) suivi d'une recomposition (Wiederzusammensetzen, Rekonstruktion) ${ }^{22}$. Dans les deux cas, l'accent est cependant mis non sur la fusion finale, concrétisant chez Kunze un " internationalisme " réel des poètes traducteurs, mais sur la phase destructrice du traducteur " contrebandier ${ }^{23}$ ". Certes, ce dernier sait que " toute poésie n'est que poursuite d'écriture du texte sans fin de la tradition ", certes, son activité est " fraternelle ", mais c'est, en tout " égoïsme " qu'il brave le regard officiel, qu'il opère au nez et à la barbe de la culture instituée, soumise au "leurre obsessionnel " (Wahnvorstellung) de l'" originalité pure ".

Le traducteur " contrebandier " est, sans scrupules, un criminel (Verbrecher) de la rupture, de l'effraction. Comme tout lecteur. Quand il n'est pas figé de respect devant les momies empoussiérées des mausolées poétiques. Dans cette perspective, la sortie des " traductions " de l'ensemble des " imitations ", qui les incluait encore dans Wasserzeichen, pourrait se lire comme l'instauration d'un écart, d'une tension bénéfique à une prise de conscience, au surgissement d'une Erkenntnis poétique. La visée deviendrait didactique, mieux, initiatique, une reprise poétique du Verfremdungseffekt brechtien.

21. Ou ces vers magnifiques d'Apollinaire, en exergue de son recueil du même nom (Le Guetteur mélancolique) :

« Et toi, mon cœur, pourquoi bas-tu / Comme un guetteur mélancolique / J'observe la nuit et la mort" -

"Und du mein Herz was pochst du noch/Ich liege, Schwermut, auf der Lauer/und lausche auf den Tod, die Nacht ".

En allemand, des assonances en " o ", puis en " a-au " répondent aux assonances françaises en " oi-a " puis en " 0 ", dont elles respectent au plus près la venue dans le vers, son battement intérieur, initié par la labiale " $\mathrm{p}$ " (pourquoi, pochst) tandis que les liquides ouvrent l'espace dans la fluidité des perceptions.

22. H.M.E., Wasserzeichen, p. VIII.

23. Enzensberger reprend d'ailleurs dans le " post-scriptum " le terme de Schmuggel quand il évoque les imitations, paraphrases et autres parodies. 


\section{L'imitation comme effraction: quand le fléau de la familiarité invite à la distanciation / Imitation als Heimbruch: wie die Heimsuchung zur Verfremdung ruft}

Kreubst du das Lerd, wo die Zertissen breun, Im dischen lurb die Gonten-Schaffeln greun, Ein sichter Wold vom bluschen Hierzel waust, Die Mespe strall und hiech der Leubahr staust, Kreubst du es wirl? Derfarn! Derfarn!

Meut ich mit dir, o mein Gebeichler, zarn. ${ }^{24}$
Kennst du das Land, wo die Zitronen blühn, Im dunkeln Laub die Gold-Orangen glühn, Ein sanfter Wind vom blauen Himmel weht, Die Myrte still und hoch der Lorbeer steht, Kennst du es wohl? Dahin! Dahin! Möcht ich mit dir, o mein Geliebter, ziehn.

Dans cette variante du grand classique goethéen, le " Mignons Lied " des Années d'apprentissage de Wilhelm Meister, l'imitation, effraction patente, traduit en langue inconnue, barbare, un texte familier à tout lecteur de langue allemande. Dans l'ambivalence de la destruction et de l'hommage s'exprime le geste fondateur de ce recueil de textes qui, son nom l'indique, ne cessent de hanter Enzensberger.

Anthologie de " contrebande ", dédiée aux « compagnons de lutte (Mitstreiter) ", les Geisterstimmen font œuvre politique de " résistance " lorsqu'elles reprennent la lutte par réfractions des Wasserzeichen ${ }^{25}$. L'enjeu: briser un regard scolaire, susciter une lecture qui serait participation active au " processus " que représente la poésie pour Enzensberger ${ }^{26}$. Un processus disjoint du processus politique, mais où le lecteur s'éprouve agent d'un processus historique plus large. Brecht n'est pas loin. De fait, par sa structure, Geisterstimmen oppose en termes de familiarité et d'" étrangèreté " deux types de jeux, deux choix de lecture-écriture. Les traductions vivifıent la poésie du fait que chaque tentative rend plus familières, plus natales (heimisch) les voix migrantes de poètes étrangers: cet accueil évite au jeu poétique de se réduire à un " sinistre match à domicile " (Heimspiel) et, rendant " la comparaison possible ", en " élève " simultanément " le niveau ". Dans cette optique d'une comparaison entre voix différentes venant d'horizons divers, aucun poème des "Traductions " n'est ici proposé en langue originale. Les imitations s'attachent à l'inverse - l'exemple introducteur l'atteste - à rendre plus étrangères, ou plutôt, à rendre audible, frappante, l'" étrangèreté " première de voix poétiques devenues si familières, si natales, qu'elles ne sont plus lues activement. L'imitation provoque. Elle suscite " étonnement et curiosité ", rappelle le caractère historique du poème, donc la dimension " éphémère " de

24. H.M.E., Geisterstimmen, p. 321.

25. Cf. Andreas Thalmayr, dans sa préface à Wasserzeichen: "Es ist etwas Geheimnisvolles an diesem zähen, stummen, entschlossenen Widerstand. Schließlich gibt es kaum etwas Harmloseres als diese dünnen Bändchen, [...] diese wohlmeinenden Anthologien. " (Wasserzeichen, p. V).

26. H.M.E. : " Poesie und Politik sind nicht Sachgebiete, sondern historische Prozesse, der eine im Medium der Sprache, der andere im Medium der Macht. Beide sind gleich unmittelbar zur Geschichte. ", in " Poesie und Politik ", Einzelheiten, Frankfurt am Main,Suhrkamp Verlag, 1962, p. 350. 
la poésie comme processus ${ }^{27}$. En ce sens, il y a bien Verfremdung brechtienne dans les imitations, qui, cette fois-ci, proposent à la comparaison et au regard critique l'original qu'elles revisitent en usant, sur le plan de la langue, d'un certain nombre de techniques éprouvées.

L'imitation-traduction d'un sonnet de Shakespeare répudie ainsi le costume d'époque d'une langue classique et endosse en toute infidélité un langage courant.

Ja, haß mich wenn du willst, doch haß mich jetzt... Then hate me when thou wilt,
$\begin{aligned} & \text { - if ever, now }-\ldots \\ & \text { Laß mich nicht lang im halben Elend schmoren! }\end{aligned}$
$\begin{array}{ll}\text { And other strains of woe, which now } \\ \text { seem woe, }\end{array}$
$\begin{aligned} & \text { Compar'd with loss of thee will not seem } \\ & \text { so. }\end{aligned}$

Contre l'énonciation apodictique et immuable à la première personne, les procédés de l'hypothèse ou de la question rhétorique rendent, avec un narrateur externe critique, impossible toute identification. Ainsi, là où le " Todtengräber " du lied de Ludwig Hölty s'exclame " Reich' und arme Leute / Werden meine Beute, / Kommen einst zu mir! ", Enzensberger questionne: "Was sind das für Leute? Wer wird deine Beute? Wer kommt einst zu dir? ".

Dans la même perspective, l'imitateur iconoclaste n'hésite pas à proposer sept variantes, de contenus très divergents, au quatrain de Brecht, Der Radwechsel. Le processus rend impossible toute interprétation monolithique d'un classique non cité, non proposé à la comparaison dans sa version originale - l'enjeu serait-il de stimuler curiosité et quête active du lecteur?

L'imitation de Goethe, du "Lied de Mignon ", citée plus haut, est certes la plus invasive, la plus stupéfiante. Tout en préservant le rythme, une destruction inédite s'opère au cœur des mots dont elle ne conserve que la dépouille, l'enveloppe des lettres initiale et finale. En effet, selon Enzensberger, toute une tradition de " compréhension " rationnelle et d'artefacts imitatifs et épigonaux a à ce point rigidifié le texte classique original que " l'incompréhensibilité première a été oubliée, refoulée, rendue inoffensive ${ }^{28}$ ". "L'aversion ", dont Goethe était alors l'objet, " est tombée dans l'oubli ${ }^{29}$ ". Un choc est nécessaire, le texte doit être rompu dans l'intimité des mots pour qu'il y ait dégel, pour qu'il recommence à battre.

27. Cf. la célèbre défınition de Bertolt Brecht: " Einen Vorgang oder einen Charakter verfremden heißt zunächst einfach, dem Vorgang oder dem Charakter das Selbstverständliche, Einleuchtende zu nehmen und über ihn Staunen und Neugier zu erzeugen [...] Verfremden heißt also Historisieren, heißt Vorgänge und Personen als vergänglich darzustellen. ", in Gesammelte Werke, Frankfurt am Main, Suhrkamp, 1967, vol. XV, p. 301, vol. XVI, p. 653.

28. Cf. H.M.E., Museum, p. 778, Vorwort 1960: "Auch Pindar und Goethe sind dunkel, nur ist diese Unverständlichkeit vergessen, verdrängt, unschädlich gemacht worden. "

29. Cf. H.M.E., "Über den Anachronismus » : " Durch die späte Kanonisation wurden sie schließlich als Klassiker installiert, und die zähe Aversion, der sie zu Lebzeiten begegneten, ist allmählich in Vergessenheit geraten. ", in Nieder mit Goethe!, Frankfurt am Main, Verlag der Autoren, 1995, p. 95. 
"Glotzt nicht so romantisch! »: chez Brecht ${ }^{30}$ ou Enzensberger, l'intimation est la même.

L'imitation œuvre ici à l'image de la hache de Kafka citée par Ingeborg Bachmann: "Ein Buch muß die Axt sein für das gefrorene Meer in uns. Das glaube ich" ${ }^{31}$ ".

Le heurt vise le choc qu'il imite. Il est piqûre de rappel de l'irruption poétique initiale. C'est la caricature intronisée que parodie ici radicalement l'imitation ${ }^{32}$.

L'imitation de Goethe devient initiation à la langue poétique, cette violence des mots au langage, à la langue-outil du quotidien, de la communication, des abus historiques ${ }^{33}$. Réduit à sa propre trace, le texte goethéen se fait brûlure, redevient intolérable pour la raison tandis que la langue use de ses sortilèges les plus puissants: libérée des obligations de communication et de compréhension immédiates, elle retrouve des qualités invocatoires perdues au quotidien. Elle déploie à travers le rythme, préservé par Enzensberger, sa puissance poétique originaire, par-delà les "significations " " incendiées " qu'évoque Octavio $\mathrm{Paz}^{34}$. La destruction (Zerbrechen) du poème fossilisé tente d'arracher ce dernier à l'impasse des regards mortifères sous lesquels la dimension classique du texte viendrait annihiler sa force poétique. L'éclatement du texte vise les œillères d'un regard que la tradition aurait insensibilisé au poétique de la langue. Au contraire, les " fragments " (Bruchstücke) de l'imitation offrent au regard désorienté un

30. Cf. le prisonnier de guerre (Heimkehrer) Kragler dans le texte de Brecht Trommeln in der Nacht (1919).

31. Ingeborg Bachmann, Frankfurter Vorlesungen, Fragen zeitgenössischer Dichtungen, München, Piper Verlag, 1980, p. 34.

32. Le procédé n'est pas sans rappeler la Oberflächenübersetzung opérée par Ernst Jandl dans le poème du même nom, où l'auteur met face à face un texte de William Wordsworth et sa propre "traduction ", réduite à une reprise, à une imitation purement phonétique (lautlich), mais avec des mots allemands, de l'original de langue anglaise. Ici aussi, il s'agit, comme le souligne le poète et germaniste autrichien Michael Hammerschmid, d'abaisser (hinunter übersetzen) le texte par sa traduction " iconoclaste " du sublime au grotesque, comme aussi de l'expulser (hinaus übersetzen) hors des " habitudes de lecture et de traduction immanentes à la littérature ". L'enjeu cependant n'est pas, chez Jandl, de réveiller le lecteur pour l'inciter à une lecture authentique de Wordsworth, mais de témoigner de son propre rapport, historique en ces années 50, et critique à un chant de la nature et à un immuable humain romantiques que la caricature nationale-socialiste rend insupportable. En ce sens, la traduction de Jandl relève, Hammerschmid le souligne très justement, moins de l'imitation en qualité de procédé que de l'attitude iconoclaste d'un " traducteur " démasquant par le jeu de "formules tragico-ridicules " les dangers de toute " esthétique élevée ". Afin de mieux se réjouir, pourrait-on ajouter, de l'impertinence irrépressible et créatrice de la langue qui ne se ferait jamais poésie qu'à rebrousse-poil, contre toute attente.

Cf. Michael Hammerschmid, "Übersetzung als Verhaltensweise ", en ligne: [http://www. engeler.de/hammerschmidluca.pdf], dernière consultation le 10 octobre 2016.

33. Dans L'Arc et la Lyre, Octavio Paz parle, c'est connu, de " déracinement ".

34. Cf. Octavio Paz, Lecture et Contemplation, p. 16: "Bien qu'on ne puisse le traduire par telle ou telle signification, ce langage n'est pas dépourvu de sens. Ou plutôt: cela même qu'il énonce ne se situe pas avant la signification, mais après. Ce n'est pas un balbutiement présignificatif: c'est une réalité à la fois physique et spirituelle, audible et mentale, qui a traversé le domaine des signifiés et les a incendiés. Elle n'est pas en deçà, mais au-delà du sens. [...] [Le dire] ne signifie rien et, en même temps, il est imprégné de sens. " 
paysage inédit, dépourvu de signification immédiate, ouvert de brèches, où un chemin vers le poème se laisserait réinventer. Le texte poétique fragmenté révèle le lied goethéen en sa nature première, l'y ramène. Ce n'est pas seulement par le contenu, trop connu, mais dans sa nature poétique même, que le poème de Goethe constitue, essentiellement, une "invitation au voyage ". Mis en regard avec le reflet imitatif d'Enzensberger, le lied se réaffırme en sa nature poétique, c'est-à-dire comme espace allégorique où résonne - ce serait le propre de tout poème - un appel au départ, au cheminement poétique à travers mille et une réfractions (Brechungen). Le lecteur y entend de nouveau ce qui, selon Ingeborg Bachmann, s'entend en toute littérature authentique: "Ein Ruf zum Aufbruch. Vielleicht zum "Utopia der Sprache 35 " ".

Entre les débris qui apparaissent là où la langue se fait mortification du mort, un espace s'ouvre au cœur des mots, par-delà les mots, là où ils ne nomment ni ne signifient plus mais se font signe, désignation d'un sens qui s'inventerait entre lecture et écriture.

Dans Geisterstimmen, les imitations créent par destruction cet espace qu'elles rendent simultanément visible. L'imitation, sur le mode pathétique ampoulé, du sobre quatrain de Rückert, " Der Tod ", rend palpable la nécessité de l'évidement, du silence: nulle présence, si ce n'est à l'écart de la dénomination. Cet écart " secret " (geheim), intérieur au poème, cet espace de jeu originel se concrétise physiquement entre l'original et son imitation, qui alors relève du contre-chant. L'écart appelle au surgir un mouvement, d'abord comparatif, qui génèrera face à l'imitation une insatisfaction programmée. Celle-ci n'a en effet pas de prétention poétique. Elle est caricaturale, car expérimentation s'annonçant comme telle: jargon, profanation, question rhétorique, néologisme, négation. Les définitions des genres imitatifs dans Wasserzeichen ou, en 2013 encore, lors d'une conférence de poétique à l'université de Tübingen, sont éloquentes ${ }^{36}$. L'enjeu des variations sur Brecht est ainsi selon Enzensberger le suivant: "Sieben Verwandlungen ..., um zu beweisen, daß das Original nicht umzubringen ist ". L'imitation de Matthias Claudius dans un style pompeux rend manifeste les dangers impliqués : " Bombast ", "Steigerung kann Schwächung bedeuten. " Sur le procédé de

35. Ingeborg Bachmann, "Literatur als Utopie ", Frankfurter Vorlesungen, p. 92 : " Die Literatur, die selber nicht zu sagen weiß, was sie ist, die sich nur zu erkennen gibt als ein tausendfacher und mehrtausendjähriger Verstoß gegen die schlechte Sprache - denn das Leben hat nur eine schlechte Sprache - und ihm darum ein Utopia der Sprache gegenübersetzt, diese Literatur [...] ist zu rühmen wegen ihres verzweiflungsvollen Unterwegsseins zu dieser Sprache und nur darum ein Ruhm und eine Hoffnung des Menschen. ". Cette langue serait langue natale, ce tourbillon benjaminien de l'origine où l'humain, en surgissant, entre dans son devenir historique et rend habitable le monde. "Ursprung ist das Ziel. " : la formule célèbre de Karl Kraus prend tout son sens dans cette perspective benjaminienne et poétique à la fois. L'homme advient à lui-même au moment où sa langue s'accomplit en revenant à sa source, en devenant " langue réalisée " (erfüllte Sprache, selon Martin Buber), chant, c'est-à-dire jeu, nous dit Rilke, nous dit Octavio Paz, jeu d'appels et de réponses.

36. Cf. Christian Gampert, " Die Lyrik ist sein Motor ", Deutschlandfunk, 21/11/2013, en ligne: [http://www.deutschlandfunk.de/poetik-vorlesung-die-lyrik-ist-sein-motor.691. de.html?dram:article_id=269729], dernière consultation le 11 novembre 2015. 
" négation " utilisé pour son imitation de Rilke, Enzensberger spécifie devant un public d'étudiants: " die Stärke dieser Stimme übersteht diesen brutalen Angriff ".

Comme dans l'art contemporain (Arthur Danto), la déception face à une installation suscite chez le lecteur l'activité qui lui donnera sens: la platitude de l'imitation revivifie la puissance de secret du texte original qui attire alors le lecteur dans son " champ magnétique " et suscite - idéalement - une lecture authentique, revivifiante.

La déception cependant n'est pas triste, l'activité est rieuse: là où le poème rend malheureux, il y a choc, savoir nouveau, Erkenntnis, espérait déjà Ingeborg Bachmann à la lecture du jeune Enzensberger ${ }^{37}$. Après avoir, en 1967, précisé sous forme d'interdit d'allégresse la sentence sur le poème écrit après Auschwitz (1951), Adorno explique comment, pour ne pas trahir le geste utopique qui les anime, l'art, le poème doivent désormais s'abstenir de rendre heureux au présent:

Stendhals Diktum von der promesse du bonheur sagt, daß Kunst dem Dasein dankt, indem sie akzentuiert, was darin auf die Utopie vordeutet. [...] Weil alles Glück am Bestehenden und in ihm Ersatz und falsch ist, muß [Kunst] das Versprechen brechen, um ihm die Treue zu halten. ${ }^{38}$

Les imitations, mais aussi les traductions, suivent chez Enzensberger ce même principe: détruire le poème afın de le revivifier et d'actualiser ainsi le processus poétique dans son enjeu utopique, donc aussi politique. Les Geisterstimmen s'avèrent le lieu non plus seulement de "l'application d'une poétique " (angewandte Poetik) mais aussi de son énonciation ${ }^{39}$. Sous couvert de jeu de masques, sous les voix de ses " compagnons de lutte " et avec elles, retentit en creux, en filigrane, sur le mode rieur, la poétique d'Enzensberger et son enjeu utopique. Dans cette poétique à la fois sienne et autre, authentique Wasserzeichen, des résonances de Goethe, d'Adorno, de Pablo Neruda notamment se fondent et se confondent en ce qui ressemblerait à une variante contemporaine, sur le mode mineur de la fracture (Bruch), de la poétique nietzschéenne, si ce n'est hesséenne de l'allégresse (Heiterkeit).

37. Le poème d'Enzensberger "Verteidigung der Wölfe gegen die Lämmer » se termine sur le vers : " ihr/ ändert die welt nicht ». Ingeborg Bachmann analyse alors : " Ja. Und das Gedicht selbst? Was bewirkt es? Ist es nicht vielleicht so, daß, weil uns so ein Gedicht unglücklich macht, weil ihm dies gelingt, [...] es auch in uns einen Ruck gibt, einen erkenntnishaften, unter dem wir den stattfindenden nachvollziehen. ", in Frankfurter Vorlesungen, p. 34.

38. Theodor W. Adorno, Ästhetische Theorie, Suhrkamp, Frankfurt am Main, 1970, p. 436.

39. Le post-scriptum l'indique à demi-mot: la critique, cette source, cette "inquiétude productive du processus poétique ", selon la deuxième des thèses de " Poesie und Politik " (1962), est, par voie de traduction, à l'œuvre dans ce recueil sous sa " forme la plus intense ". 


\section{Faut-il rompre la promesse du bonheur? Du politique à l'utopique, le grand sérieux du rire}

La poétique d'Enzensberger apparaît, de manière larvée, à travers les quatre seules imitations du recueil qui portent cette dénomination et qui, de surcroît, vont rompre l'ordre chronologique affiché. Celles-ci sont pures démonstrations du politique du poétique: elles sont politiquement résistantes, c'est-à-dire insaisissables. On comprend mieux l'absence, face aux sept variations, de l'original brechtien sur lequel il reste impossible - et Enzensberger en fait la démonstration par l'exemple - de mettre la main. Ne cite-t-il pas, depuis 1962, Der Radwechsel en exemple quand il s'agit de défınir le politique du poétique, le geste politique du poème? " Das Gedicht [Der Radwechsel] spricht mustergültig aus, daß Politik nicht über es verfügen kann: das ist sein politischer Gehalt ${ }^{40}$ ".

Préserver ce " noyau du processus poétique " est vital sur le plan humain en ce qu'il relève, comme chez Adorno, mais aussi chez Hesse ou Nietzsche, de l'utopique, en ce qu'il a trait au bonheur. C'est d'ailleurs sur le terme de Glück que s'achève à la fois l'article "Poésie et politique " et le premier recueil d'essais d'Enzensberger Einzelheiten, non sans avoir d'abord spécifié ce que vise le dire poétique: "Poesie tradiert Zukunft. Im Angesicht des gegenwärtig Installierten erinnert sie an das Selbstverständliche, das unverwirklicht ist ${ }^{41}$ ".

$\mathrm{Si}$ " la fonction politique du poème est de se refuser à toute fonction politique ", si la " critique " prend la relève de l'inspiration pour devenir moteur, " inquiétude productive du processus poétique ", c'est uniquement dans la tension et la concomitance entre " critique " et " anticipation " qu'il y a surgissement poétique, que se dessine un chemin où le " bonheur " deviendrait possible.

Cette anticipation peut prendre les traits " du doute, du refus, du non ». En cela, Enzensberger reste proche d'un Adorno rompant la promesse de bonheur inhérente à l'art dans le souci d'en préserver la dimension utopique. Mais l'humour qui conduit Enzensberger à escamoter l'original brechtien de ses imitations montre qu'il déploie le thème de la fracture, des plus présents dans les quatre imitations clés, sur un mode " autre " qui est le sien, non agréé, du rire iconoclaste et séducteur à la fois.

Ces quatre "imitations " ressembleraient presque à un manifeste de ce politique du poétique. Le compositeur Hans Werner Henze ne s'y est pas trompé en plaçant trois d'entre elles à la fin de son cycle de Lieder Voices (! ) (1971), où il témoignait, à ses propres dires, " de sa pensée politique personnelle et de son engagement politique émotionnel ${ }^{42}$ ". Das Blumenfest, imitation aztèque, qui concluait le cycle de Henze, ouvre ici celui des imitations, tandis que l'avant-dernier poème du cycle, Schluß, imitation de Michalis Katsaros, clôt le

40. H.M.E., " Poesie und Politik », p. 350.

41. Ibid., p. 353.

42. Hans Werner Henze: "Die Auswahl der Gedichte und ihre Anordnung reflektieren mein persönliches politisches Denken und mein emotionelles politisches Engagement. ", en ligne: [http://www.hans-werner-henze-stiftung.de/hans-werner-henze/werkverzeichnis/detail/ news/detail/News/stimmen-voices/], dernière consultation le 11 novembre 2015. 
recueil de Geisterstimmen. Les fleurs, ce sont explicitement les mots, des offrandes destructrices et enchanteresses. Et la fin est nécessaire au début, à l'Aufbruch: "Wenn wir mit alledem Schluß gemacht haben / können wir anfangen ".

Quant au poème Puzzle, l'avant-dernier du recueil, est-il nécessaire d'en souligner les ruptures constitutives, quand l'imitation du texte de Karl Schippers s'attache à rompre en puzzle, non les "tableaux " de la version originale, mais des " poèmes " accrochés sur des murs de " musées "? Ce sont d'ailleurs ces trois dernières imitations, regroupées, (Brecht-Schippers-Katzaros) qui mettent en désordre l'ordre chronologique des autres imitations, tout comme l'ensemble des imitations remet en cause leur distinction apparente d'avec les traductions. Les frontières sont fluctuantes, les lignes de rupture en jeu incessant.

De fait, sur le mode de la fracture, les imitations mettent en rire l'ensemble des traductions, ce bouquet de fleurs différent, ce musée personnel, subjectif, d'une poésie autre, qui est aussi celle d'Enzensberger: une poésie de l'obscur, des petites choses, de l'ici-bas, de l'humble. Ou, pour le dire avec la " métaphore centrale " de l'œuvre de jeunesse de Pablo Neruda, une poésie du " el roto ", de ce qui est "brisé " (zerbrochen) $)^{43}$, de ce qui " souillé ", " usé " est " la substance même du monde ", selon les mots d'Enzensberger dans son essai de 1955, alors que son premier recueil, Verteidigung der Wölfe, n'était pas encore paru (1957). C'est dire si Neruda est à la source de la poésie d'Enzensberger. Les poèmes de Neruda se retrouvent d'ailleurs à la fois parmi les traductions et les imitations de Geisterstimmen. Plus précisément, le poème « Melancolia en las familias ", déjà cité en 1955, est le seul à être d'abord présenté comme traduction puis, dans la seconde partie, en vis-à-vis d'une imitation iconoclaste " Agonie in den Kleiderschränken ».

Chez Enzensberger, fracture, rupture, destruction - la " mélancolie " même s'écrivent, on le voit, sur le mode du rire. Un rire très nietzschéen, voire zarathoustrien, comme dans un poème clé de Verteidigung der Wölfe (gegen die Lämmer!):

Es herrscht ein Mangel an Männern,

das Aussichtslose tuend stumm,

ausraufend wie Gras die Hoffnung,

ihr Gelächter, die Zukunft, rollend,

rollend ihren Zorn auf die Berge.

Tout le champ lexical autour du rire, "froid ", incisif, destructeur, est de tonalité nietzschéenne, tout comme la joie associée à la destruction et l'autoportrait en contrebandier ou criminel. L'élégie de Rilke pourrait n'avoir été imitée, retournée, détournée par Enzensberger sous forme de « négation » que pour faire résonner ce vers:

Kein Engel ist schrecklich.

Und so halt ich nicht länger an mich und lasse den

Lockruf

hellen Gelächters laut werden. ${ }^{44}$

43. Il s'agit du Neruda de la première période, avant qu'il ne cède, selon Enzensberger, au leurre de la poésie comme instrument de la politique. Les poèmes repris datent d'ailleurs exclusivement de cette période et sont loin des transparences de la propagande socialiste.

44. L'original de Rilke disait: «Ein jeder Engel ist schrecklich. / Und so verhalt ich mich denn und verschlucke den / Lockruf / dunkelen Schluchzens », in H.M.E., Geisterstimmen, p. 344. 
Se retrouve ici toute une poétique de l'allégresse jouant de flèches de désir (Sehnsucht) et de destruction ${ }^{45}$. Contrairement au diagnostic adornien, allégresse (Heiterkeit) et légitimité ne sont pas incompatibles pour Enzensberger. Ce dernier semble bien plus imiter la stratégie de légitimation nietzschénne dans les préfaces de 1886 en adoptant dans les "préface " et " post-scriptum " un ton rieur, mais aussi en soulignant le plaisir (Vergnügen) trouvé à lire, écrire, traduire, jusqu’à l'" addiction " (Sucht). Le risque étant d'achopper au même écueil de justification dans une perspective de Heilsrelevanz. Le mouvement premier cependant, celui de Sisyphe, " das Aussichtslose tuend ", relève bien de l'humour hesséen, incarné par celui qui, tel Confucius, sait que c'est impossible mais le fait quand même ${ }^{46}$.

Plus généralement, la tension entre " critique " et " anticipation " dont se nourrit le processus poétique selon Enzensberger semble reconduire, dans une perception du politique et de l'utopique similaire ${ }^{47}$, la bipolarité de " rappel " (Mahnung) et de "consolation " (Trost) propre à la poétique de l'allégresse hesséenne. Bipolarité qui reflète l'ambivalence première de la Heiterkeit entre hilarité iconoclaste, dionysiaque, et sérénité apollinienne. Dans cette perspective, Geisterstimmen réactualise, dans sa structure même, une tension essentielle au processus poétique, à une initiation à la lecture et à la relecture dans un mouvement infıni et indéfıni. Contre toute rigidification muséale, les Imitations remettent en jeu, rappellent au rire les poèmes de l'obscur et de l'absence des Traductions. Reconnaissance et offrande de ces dernières permettent à l'effervescence ludique des Imitations de ne pas se perdre en virtuosité érudite, tandis que leur sombre rayonnement attire au secret de leur grand sérieux les plus donquichottesques des lecteurs : là où, peut-être, à la source du geste créateur, l'humour rejoindrait l'utopique et le politique.

Le parallèle avec Reiner Kunze apparaît alors comme fructueusement antipodique, Enzensberger et Kunze jouant, dans un refus identique d'une politisation du poétique mais dans des contextes contraires, sur des modes mineur et majeur opposés les deux pôles d'une poétique de l'allégresse. L'évocation de

45. Cf. Béatrice Poulain, " Poétique de l'allégresse. Initiation à la Heiterkeit dans l'œuvre en prose d'Hermann Hesse ", en ligne: [http://www.e-sorbonne.fr/theses/poetique-l-allegresseinitiation-heiterkeit-l-oeuvre-prose-d-hermann-hesse].

46. On ne s'étonnera pas qu'Enzensberger ne mentionne guère Hesse - le citer est de nos jours de mauvais aloi - hormis au détour d'un Schüttelreim ironique et sous le pseudonyme d'Andreas Thalmayr: "Man sagt sich: Ich vergesse halt, / wie viel mir Hermann Hesse galt.». De fait, la manière de souligner la dimension subjective du choix des poèmes dans Geisterstimmen réveille en échos l'essai La Bibliothèque universelle. Certaines tournures et mots, " die sogenannte Wirklichkeit ", Eigensinn, ont également des consonances hesséennes. Pour leur part, Heiterkeit et humour - restés suspects - sont seulement présents, et donc d'autant plus en filigrane, sous le masque de Goethe, ou dotés en outre de consonances thomas manniennes. Dans un entretien au sujet de son montage radiophonique " Nieder mit Goethe! ", sous-titre " eine Liebeserklärung ", Enzensberger explique ainsi combien il admire surtout la " souveraineté, la nonchalance, l'humour si l'on peut dire (!) " de Goethe. L'imitation du poème de Mignon paraissait, elle, dans Wasserzeichen sous un pseudonyme évoquant fortement Doktor Faustus, l'anagramme Serenus Brezengang.

47. Le prétendu revirement apolitique d'Enzensberger fin des années 60 apparaît alors comme un approfondissement méconnu du politique. 
Sisyphe est ici emblématique. Au caractère destructeur d'Enzensberger répond, chez Kunze, la consolation, au dionysiaque, l'apollinien :

Die Kunst verspricht Sisyphos nichts und versucht nicht, sein Selbstverständnis zu brechen und sein Denken zu unterwerfen, damit er zu seinem Heil gelange, sondern macht ihn seinem Schicksal gegenüber widerstandsfähiger. ${ }^{48}$

Là où Enzensberger récuse, par souci d'obscur et de terrestre, le beau, l'internationalisme du marché mondial globalisé et opte pour l'imitation, contre les interprétations poétiques trop monolithiques et asphyxiantes, Kunze, ardent partisan de la lumière, défend le beau contre les mises en demeure idéologiques, oppose à l'internationalisme hypocrite du socialisme étatique l'internationalisme fécond des poètes traducteurs et se joue, par déconstruction, des interprétations aux " concepts éclairants " qui accroissent la joie générée par le texte. Là aussi, des brèches s'ouvrent, un espace se crée où l'obscur devient offrande de lumière, et l'éclair, rappel de la terre.

Deux poèmes d'Enzensberger et de Reiner Kunze sonneraient presque comme une invitation à une telle lecture :

Hans Magnus Enzensberger ${ }^{49}$

\section{KOPFKISSENGEDICHT}

Dafür, daß du bis in die Fingerspitzen anwesend bist, daß es dich verlangt, dafür, wie du die Knie biegst und mir dein Haar zeigst, für deine Temperatur und deine Dunkelheit ; für deine Nebensätze, das geringe Gewicht der Ellenbogen und die materielle Seele, die in der kleinen Mulde über dem Schlüsselbein schimmert ; dafür, daß du gegangen und gekommen bist, und für alles, was ich nicht von dir weiß, sind meine einsilbigen Silben zuwenig, oder zuviel. 
Reiner Kunze ${ }^{50}$

WIE DIE DINGE AUS TON

Aber ich klebe meine hälften zusammen wie ein zerschlagener topf aus ton. Jan Skacel, brief vom februar 1970

1.

Wir wollten sein wie die dinge aus ton

Dasein für jene,

die morgens um fünf ihren kaffee trinken in der küche

$\mathrm{Zu}$ den einfachen tischen gehören

Wir wollten sein wie die dinge aus ton, gemacht aus erde vom acker

Auch daß niemand mit uns töten kann

Wir wollten sein wie die dinge aus ton Inmitten

soviel rollenden stahls

2.

Wir werden sein wie die scherben der dinge aus ton: nie mehr ein ganzes, vielleicht ein aufleuchten im wind

50. Reiner Kunze, " wie die dinge aus ton ", in zimmerlautstärke (1972), in Gedichte, Frankfurt am Main, Fischer Verlag, 2007, p. 109. 
